3. La sous-commission, prenant en considération le plan de travail étendu, suggéré par le Dr Whipple, estime qu'elle doit être tenue au courant de tous les progrès réalisés dans l'étude de la haute atmosphère, quelles que soient les méthodes utilisées.

Elle souhaite qu'à cet effet une liaison étroite soit établie entre elle et les commissions de l'Union Géophysique Internationale et de l'U.R.S.I., s'occupant de problèmes connexes.

\title{
Commission 23. (CARTE DU CIEL)
}

I. L'Assemblée Générale de l'U.A.I. et la Commission de la Carte du Ciel attirent l'attention des Gouvernements du Commonwealth et des Etats Australiens sur l'importance qui s'attache à l'achèvement des zones du Catalogue Photographique du Ciel confiées aux observatoires australiens; elles leur demandent d'en favoriser l'exécution en programme d'urgence.

Les dernières lacunes du Catalogue Photographique nuisent à l'utilisation globale de cet instrument scientifique précieux, qui a demandé tant d'efforts internationaux; elles sont d'autant plus regrettables que l'essentiel du travail a déjà été fourni. L'U.A.I. serait reconnaissante à Monsieur H. W. Wood, Government Astronomer, Sydney, New South Wales, d'accepter la direction de cet achèvement et à Monsieur le Dr R. v. d. R. Woolley, Directeur de l'Observatoire du Commonwealth, Canberra, de lui fournir les aides nécessaires.

La Commission de la Carte du Ciel s'engage à demander l'aide financière de l'U.A.I. pour la publication des derniers volumes, lorsque les manuscrits seront prêts pour l'impression.

2. La Commission de la Carte du Ciel, désireuse de voir se terminer la publication du Catalogue Photographique, demande à l'U.A.I. de mettre à sa disposition, en plus des fonds qui lui ont été attribués et qui ne sont pas encore dépensés, une somme de vingt-et-un mille francs-or pour couvrir les frais d'impression de 5 volumes du Catalogue, appartenant aux zones laissées en souffrance par les Observatoires de Potsdam, de Perth et de Rio-deJaneiro et dont les manuscrits, préparés par les Observatoires d'Oxford et d'Edimbourg, pourraient être imprimés d'ici I95I.

\section{Commission 24. (PARAllaXes Stellaires et Mouvements PROPRES)}

La Commission recommande que le Comité Exécutif soit prié d'allouer un montant de \$1500 pour les frais d'impression et de reliure de la troisième édition du General Catalogue of Parallaxes. L'Observatoire de Yale a entrepris la compilation et la préparation du manuscrit dactylographié pour l'impression.

\section{Commission 27. (ÉTOILES VARIABLES)}

I. La Commission recommande que de petites cartes soient publiées pour chaque étoile variable nouvellement découverte, quand cette étoile ne figure dans aucun catalogue méridien.

2. La Commission recommande qu'une subvention de $\$ 250$ soit mise à la disposition de son président pour rétribuer un dessinateur qui serait chargé de préparer des cartes d'étoiles variables australes pour les astronomes amateurs.

3. La Commission tient à mettre en relief l'importance des observations visuelles de certaines classes d'étoiles variables.

4. La Commission approuve les trois résolutions proposées par MM. H. N. Russell, J. E. Merrill et N. L. Pierce:

$I^{\circ}$. Les époques des minima des variables à éclipses doivent être publiées individuellement, en Temps Moyen de Greenwich, c'est-à-dire, en faisant commencer le jour à midi moyen de Greenwich. Ces époques seront héliocentriques, et exprimées en jours et fractions décimales du jour. En aucun cas, la publication ne pourra être limitée à celle d'une époque normale. Si l'on veut donner les résidus des observations par rapport à une 
éphéméride, on fournira le résidu correspondant à chaque minimum observé, ainsi que la formule numérique ayant servi au calcul de l'éphéméride.

$2^{\circ}$. Pour rémédier autant que possible au manque d'observations pendant les dernières années, il est recommandé de déterminer, au cours des deux ou trois années à venir, les minima de toutes les variables à éclipses intéressantes, non seulement de celles qui offrent un mouvement de la ligne des apsides, mais encore de celles dont la période semble présenter des fluctuations. Les observations visuelles peuvent rendre ici de grands services.

$3^{\circ}$. L'étude détaillée des systèmes binaires à éclipses les plus intéressants exigeant la connaissance de leurs variations spectrales, il est recommandé de mener autant que possible simultanément les observations photométriques et les observations spectrographiques. Les unes et les autres devront être concentrées sur le plus court intervalle de temps possible. La même recommandation s'applique aussi à l'étude des céphéides brillantes, pour lesquelles on peut obtenir des spectrogrammes à grande dispersion.

5. La Commission signale l'importance des observations spectrographiques d'étoiles variables dans l'hémisphère sud.

6. La Commission a approuvé les règles pour la dénomination des étoiles variables, qui ont été provisoirement adoptées par la sous-commission de la dénomination des étoiles variables.

Ces règles sont:

Doivent être dénommées:

(a) les variables périodiques dont le type, la période et la courbe de lumière sont connus;

(b) les variables non périodiques dont le type a été bien établi;

(c) les variables dont le type paraît être évident, bien qu'aucun autre détail ne soit donné.

Le choix des étoiles, satisfaisant aux règles mentionnées ci-dessus, doit reposer sur une discussion stricte de toutes les données.

7. La Commission propose que la subvention annuelle accordée à M. Banachiewicz pour la publication des éphémérides des étoiles doubles à éclipses, soit continuée à raison de rooo (mille) francs-or, à la condition que les dates soient données exclusivement dans la période julienne.

8. La Commission a décidé de demander à l'Assemblée Générale de faire imprimer et distribuer une traduction en anglais de l'introduction, de la liste des auteurs russes et des remarques du nouveau catalogue général des étoiles variables, publié par MM. Kukarkin et Parenago à la demande de 1'U.A.I. Le nombre des pages données en langue et caractères russes est d'environ vingt. Une subvention unique de 250 dollars serait nécessaire probablement.

9. La Commission a décidé de demander à l'Assemblée Générale de faire imprimer et distribuer la 44ième liste des noms d'étoiles variables, dont le manuscrit a été préparé par MM. Kukarkin et Parenago. La liste contient la dénomination de 266 étoiles variables nouvelles. Une subvention annuelle de 250 dollars suffira probablement pour couvrir les frais.

I0. La Commission demande que le Bureau de l'Union entreprenne la publication de la liste des observations inédites des étoiles variables, dont M. Grouiller avait été chargé en r938. M. Brun a communiqué que le manuscrit se trouve à l'Observatoire de Lyon prêt pour la publication.

II. La Commission approuve la formation d'une commission nouvelle (42) sur les étoiles doubles photométriques.

\section{Commission 28. (NÉBULEUSES EXTRA-GALACTIQUES)}

La Commission attire l'attention sur le fait que les remarquables photographies de nébuleuses faites par le docteur C. O. Lampland de l'Observatoire Lowell n'ont pas encore 